\title{
Modulation of motor cortex excitability after upper limb immobilization
}

\author{
Giampietro Zanette $^{\mathrm{a}, *}$, Paolo Manganotti ${ }^{\mathrm{b}}$, Antonio Fiaschi ${ }^{\mathrm{b}}$, Stefano Tamburin $^{\mathrm{a}, \mathrm{b}}$ \\ ${ }^{a}$ Dipartimento di Scienze Neurologiche e della Visione, Sezione di Neurologia, Ospedale Policlinico G.B. Rossi, piazzale Scuro, 37134 Verona, Italy \\ ${ }^{\mathrm{b}}$ Section of Neurological Rehabilitation, Department of Neurological Sciences and Vision, University of Verona, Italy
}

Accepted 30 December 2003

\begin{abstract}
Objective: To examine the mechanisms of disuse-induced plasticity following long-term limb immobilization.

Methods: We studied 9 subjects, who underwent left upper limb immobilization for unilateral wrist fractures. All subjects were examined immediately after splint removal. Cortical motor maps, resting motor threshold (RMT), motor evoked potential (MEP) latency and MEP recruitment curves were studied from abductor pollicis brevis (APB) and flexor carpi radialis (FCR) muscles with single pulse transcranial magnetic stimulation (TMS). Paired pulse TMS was used to study intracortical inhibition and facilitation. Compound muscle action potentials (CMAPs) and $F$ waves were obtained after median nerve stimulation. In 4/9 subjects the recording was repeated after 35-41 days.

Results: CMAP amplitude and RMT were reduced in APB muscle on the immobilized sides in comparison to the non-immobilized sides and controls after splint removal. CMAP amplitude and RMT were unchanged in FCR muscle. MEP latency and F waves were unchanged. MEP recruitment was significantly greater on the immobilized side at rest, but the asymmetry disappeared during voluntary muscle contraction. Paired pulse TMS showed an imbalance between inhibitory and excitatory networks, with a prevalence of excitation on the immobilized sides. A slight, non-significant change in the strength of corticospinal projections to the non-immobilized sides was found. TMS parameters were not correlated with hand dexterity. These abnormalities were largely normalized at the time of retesting in the four patients who were followed-up.

Conclusions: Hyperexcitability occurs within the representation of single muscles, associated with changes in RMT and with an imbalance between intracortical inhibition and facilitation. These findings may be related to changes in the sensory input from the immobilized upper limb and/or in the discharge properties of the motor units.
\end{abstract}

Significance: Different mechanisms may contribute to the reversible neuroplastic changes, which occur in response to long-term immobilization of the upper-limbs.

(C) 2003 International Federation of Clinical Neurophysiology. Published by Elsevier Ireland Ltd. All rights reserved.

Keywords: Plasticity; Immobilization; Sensorimotor restriction; Transcranial magnetic stimulation; Cortical hyperexcitability; Motor system

\section{Introduction}

Evidence from animal and human experiments suggests that neuroplastic changes may occur in sensorimotor areas of the adult nervous system following modification of somatic afferences or of motor output (Jones, 2000; Sanes and Donoghue, 2000). Learning new motor skills (Pascual-Leone et al., 1995) and performing skilled motor activities (Elbert et al., 1995) result in an expansion of the representation of the muscles involved in the task. Complete long term sensorimotor deafferentation, as in the case of limb amputation (Chen et al., 1998; Cohen et al., 1991; Kew et al., 1994; Ridding and Rothwell, 1997; Wu and Kaas,

\footnotetext{
* Corresponding author. Tel.: + 39-045-6449132; fax: + 39-045-585933 E-mail address: gi.zanette@libero.it (G. Zanette).
}

1999) and peripheral nerve lesions (Rijntjes et al., 1997; Tinazzi et al., 1998), as well as short term deafferentation secondary to ischemic nerve block (Brasil-Neto et al., 1993; Ridding and Rothwell, 1997; Ziemann et al., 1998a; Ziemann et al., 1998b), result in an expansion of the surrounding representations.

While a great deal of experimental evidence has been gathered on neuroplasticity following sensorimotor deafferentation, little is known about the changes taking place after limb disuse. This condition is associated with sensorimotor restriction, which is functionally different from the complete deafferentation following nerve lesions or limb amputation. Animal studies suggest that sensory impoverishment may determine changes in the organization and size of cortical receptive fields in the somatosensory 
cortex (Coq and Xerri, 1999; Langlet et al., 1999). The vast majority of studies on the effects of long term immobilization in humans have examined changes in the contractile properties of skeletal muscle (Desaphy et al., 2001; Seki et al., 2001b) or motor units (Seki et al., 2001a). Only two reports to date have documented central motor changes in neurologically normal subjects who wore splints for more than four weeks because of fractures of the wrist (Zanette et al., 1997) or the leg (Liepert et al., 1995). These two transcranial magnetic stimulation (TMS) studies yielded conflicting results, because the latter found a reduction in the motor maps of immobilized muscles, while the former reported maps of normal area, but greater responses to TMS. Data from stroke patients, treated with constraint-induced therapy (Taub et al., 2002) are able to add little information on the topic, because motor stroke per se is capable of inducing very extensive bilateral cortical reorganization (Liepert et al., 2000).

The experimental model of limb disuse offers a unique opportunity to evaluate the changes taking place within the representation of the immobilized body parts. Most of the studies on neuroplasticity have been conducted on surviving body parts or nerves and can provide only indirect information on the changes occurring in the deprived cortical areas. To date there has been only one report evaluating the functional correlates of somatosensory reorganization within the representation of injured nerves (Moore and Schady, 2000).

In an attempt to better understand the mechanisms of motor changes after long term sensorimotor restriction, we applied TMS to nine patients, whose left upper limbs were immobilized for wrist fractures. The study was aimed at elucidating different points. The first was to evaluate the contribution of the motor cortex in the generation of motor hyperexcitability. The second was to understand whether the changes in motor maps represent true representational plasticity or the presence of a different level of 'rest' of the corticospinal system (Siebner and Rothwell, 2003). The third point was to test if any change took place in the representation of the unrestricted limb. Finally we were interested in establishing a correlation between indices of motor excitability and hand function. It is common experience that hand movements are clumsy after splint removal. Even though mechanical factors, such as stiffness of joint and muscle and the lack of motor practice play a role in worsening hand dexterity, cortical changes may contribute to the clumsiness, due to the importance of the motor cortex in fine hand movements. To these aims, we studied resting motor threshold (RMT) in response to TMS, motor cortical maps, TMS recruitment curves, intracortical inhibition (ICI) and facilitation (ICF) and F waves in our patients. In this experimental setting we could not obtain baseline (prior to fracture) data for the patients, thus we compared immobilized vs normal sides of patients and patients vs normal controls. The possible correlation between the abnormalities and hand dexterity was studied.
Patients were re-tested 5-6 weeks later to understand to which extent the changes were reversible.

\section{Materials and methods}

\subsection{Subjects}

We studied 9 patients (4 males, 5 females), aged 18-52 years (mean age $36.9 \pm 11.7$ years), who wore splints for traumatic fractures of the left wrist. None of them underwent surgical treatment of the fracture. None of the patients had any history of neurological disease. The mean duration of immobilization was $37.4 \pm 4.4$ days (range $30-45$ days). The splints immobilized the wrist, elbow and metacarpophalangeal joints and the upper limb was fixed in a midrange flexion-extension position. There was no restriction of the scapulo-humeral joint, but the splint forced the patients to maintain preferential adduction of the upper limb. The patients were able to perform partial flexion-extension movements of the distal parts of the digits of the hand. Pain sensations related to the traumatic fractures were referred only during the first 8-13 days of immobilization. At splint removal, the patients presented slight muscular atrophy and hyposthenia in the affected arm. No sensory disturbances were referred, and the neurological examination findings were unremarkable. A standard nerve conduction and electromyographic study was performed in all patients to exclude the presence of peripheral nerve lesions secondary to the trauma or to wearing the splint. All patients were studied immediately after splint removal (T0). Recording was repeated 35-41 days after splint removal (T1) in 4 patients. Twelve healthy age-matched subjects (6 males, 6 females), aged 20-48 years (mean age 35.8 \pm 9.4 years) volunteered as controls. All patients and controls were righthanded, according to the Edinburgh inventory (Oldfield, 1971). Local hospital ethical committee approval was obtained and all subjects gave their written informed consent for the study. Digital dexterity was evaluated by measuring the time taken to pick up 10 metal pegs from a cup and insert them one by one in a row of holes (Purdue pegboard test; Van der Kamp et al., 1991).

\section{2. $M E P$ and CMAP recording}

Motor evoked potentials (MEPs) were recorded using pairs of $\mathrm{Ag}-\mathrm{AgCl}$ surface electrodes taped on the belly and tendon of the abductor pollicis brevis (APB) and flexor carpi radialis (FCR) muscles of both sides of normal controls and on the immobilized and normal sides of patients. EMG signals were filtered (bandpass $5 \mathrm{~Hz}$ to $20 \mathrm{KHz}$ ), amplified, displayed (Dantec Keypoint, Medtronic, Skovlunde, Denmark) and stored for off-line analysis. Peak-to-peak amplitudes and latencies of MEPs were measured.

Compound muscle action potentials (CMAPs) were determined by supramaximal electrical stimulation of 
the median nerve at the wrist for the APB and above the elbow for the FCR.

\subsection{Transcranial magnetic stimulation}

All subjects wore ear-plugs during the experiments and were seated in an armchair with the elbow semiflexed and the forearm pronated, fully relaxed and supported by the arm of the chair. All subjects were invited to be fully relaxed during TMS. Magnetic shocks were delivered with a Novametrix Magstim 200 (The Magstim Co. Ltd, Whitland, UK) magnetic stimulator. TMS was performed with a circular coil (outer diameter $130 \mathrm{~mm}$ ) and a figure-of-eight focal coil (outer diameter of each loop $87 \mathrm{~mm}$; peak magnetic field $2.2 \mathrm{~T}$ ) adjusted over the optimal scalp position to evoke the maximal MEP amplitude in each target muscle (Rossini et al., 1994). The resting motor threshold (RMT) were defined as the lowest stimulator output intensity capable of inducing MEPs of at least $50 \mu \mathrm{V}$ peak-to-peak amplitude in the target muscle(s) in at least half of 10 trials (Rossini et al., 1994). The RMT was determined by varying the stimulator intensity in $1 \%$ steps. The current direction in the circular coil was anticlockwise when viewed from above (side A) for preferential left motor cortex activation and clockwise (side B) for the right motor cortex. The handle of the figure-of-eight focal coil was pointed occipitally so that the current in the brain flowed in a posterior to anterior direction so as to activate corticospinal neurons mainly transsynaptically (Werhahn et al., 1994). The coil position was marked on the scalp and its consistency was continuously monitored throughout the experiment. Both visual feedback from an oscilloscope and audio feedback were used to ensure that the recorded muscle was relaxed.

\subsection{Motor cortex mapping}

A thin elastic cotton cap was placed on the scalp of the subjects. The cap had a grid of multiple stimulation points, marked $1 \mathrm{~cm}$ apart, in both the coronal and sagittal planes with reference to Cz. Maps of the APB and FCR muscles were obtained by delivering four stimuli at each position over the contralateral hemisphere at an intensity $30 \%$ above the RMT of the FCR muscle. The peak-to-peak amplitudes of four MEPs recorded for each muscle over each scalp position were averaged off-line and expressed as a percentage of CMAP $(\% \mathrm{M})$. The area of the map was defined as the number of scalp positions at which MEP could be elicited and the volume of the map was defined as the sum of the \%M at each point (Zanette et al., 1997). The center of gravity $(\mathrm{CoG})$ of the map was calculated as an $x-y$ coordinate from the distribution of response amplitude, according to previous authors (Röricht et al., 2001).

\subsection{MEP recruitment curve}

MEP recruitment was studied with the circular coil at rest and during slight voluntary contraction. The stimulus intensities studied were 100, 110, 120, 130, 140 and $150 \%$ of the RMT for each muscle. Eight pulses were delivered for each stimulus intensity, with stimulus intensities administered randomly. To avoid collecting startle or reflex responses, we excluded the first MEP for each trial from the analysis. The peak-to-peak amplitudes of the MEPs recorded from each muscle for each stimulus intensity were averaged and expressed as \% $\mathrm{M}$.

\subsection{Paired pulse TMS}

Intracortical excitability was studied using paired stimulus paradigms to investigate intracortical inhibition and facilitation (Kujirai et al., 1993). For this purpose, two magnetic stimulators were connected to a focal coil through a Bistim device (The Magstim Co., UK). The subthreshold conditioning stimulus was set at $70 \%$ the RMT and was delivered through the same magnetic coil at interstimulus intervals (ISIs) of 1, 2, 3, 4, 5, 7, 10, 15, 20 and $30 \mathrm{~ms}$ before the suprathreshold test stimulus (110-120\% RMT). The test stimulus was set in order to evoke a test MEP amplitude of comparable size $(0.5-1 \mathrm{mV})$ in all subjects. Eight responses were recorded for each ISI and different ISIs were applied randomly. To avoid collecting startle or reflex responses, we excluded the first conditioned MEP for each trial from the analysis. For each ISI the MEPs peak-to-peak amplitudes were averaged and expressed as a percentage of the unconditioned response. Intracortical inhibition $\left(\mathrm{ICI}_{2-4}\right)$ and intracortical facilitation ( $\left.\mathrm{ICF}_{10-30}\right)$ were calculated by averaging the ratios across ISIs of $2-4$ and $10-30 \mathrm{~ms}$, respectively.

\section{7. $F$-wave recording}

Spinal excitability was assessed by F-wave recording. $\mathrm{F}$ waves were recorded in the APB muscle in response to median nerve stimulation at the wrist. $F$ waves were obtained from 32 supramaximal stimuli at $1 \mathrm{~Hz}$ frequency and amplified using a band-pass of $100 \mathrm{~Hz}-5 \mathrm{KHz}$. The following F-wave parameters were evaluated: persistence (ratio of number of $\mathrm{F}$ waves obtained to the number of electrical stimuli, expressed as a percentage), minimal latency, mean amplitude, mean area, F-wave to CMAP amplitude ratio.

\subsection{Statistical analysis}

Analysis of variance (ANOVA) and post-hoc analysis with Bonferroni's correction were used for the comparisons of digital dexterity, CMAP amplitude, RMT, MEP latency, MEP recruitment curves, paired TMS data, $F$ waves in relation to the side (immobilized and free side of patients, 
left and right side of controls). Side-to-side comparisons of area, volume and CoG of cortical maps were made using the paired $t$ test, which was also used to compare different measures at $\mathrm{T} 0$ and $\mathrm{T} 1$ in the patients followed up. The correlation between indices of motor excitability and the duration of the immobilization was tested with the Pearson $r$-coefficient. The correlation between indices of motor excitability and hand function was examined in patients at T0 and T1 with the Spearman's correlation test. $P<0.05$ was taken as the significance threshold.

\section{Results}

ANOVA showed a significant effect of the side on manual dexterity $[F(3,44)=156.7, P<0.001]$. No differences were found between the right $(14.5 \pm 3.2 \mathrm{~s})$ and left sides $(15.6 \pm 4.2 \mathrm{~s})$ of normal controls and the free sides of patients $(15.1 \pm 4.5 \mathrm{~s})$. The patients took significantly longer to complete the test on the immobilized side $(34.4 \pm 5.7 \mathrm{~s} ; P<0.001)$.

Significant reductions in CMAP amplitude $[F(3,44)=4.8$, $P<0.01$; patients' immobilized sides vs controls' left sides, $P=0.02$; patients' immobilized vs free sides, $P<0.02)]$ and RMT to circular coil $[F(3,44)=4.3, P=0.01$; patients' immobilized sides vs controls' left sides, $P=0.02$; patients' immobilized vs free sides, $P=0.02)]$ were found in APB muscle but not in FCR muscle [CMAP $F(3,44)=2.2 ;$ RMT $F$ $(3,44)=2.6$; Table 1]. MEP latency did not differ significantly between sides $[F(3,44)=0.9$; Table 1$]$.

\subsection{Motor cortical maps}

No side-to-side differences were found in area, volume or CoG of motor cortical maps in normal subjects $(n=5$; Table 2). No side-to-side differences were found in area or CoG in patients ( $n=4$; Table 2$)$. Volume was significantly larger on immobilized sides as compared to free sides of patients $(P<0.001$; Table 2; Fig. 1).

\subsection{MEP recruitment curves}

MEP amplitude at rest was greater on the immobilized sides as compared to free sides of patients and to normal controls at all TMS intensities in both APB (Fig. 2A) and FCR muscles (Fig. 2C). Repeated measures ANOVA (between-subjects variable: side; within-subjects variable: TMS intensity) showed a significant effect of the side [APB $F(3,38)=21.8, \quad P=0.0001 ;$ FCR $F(3,38)=13.4$, $P=0.002]$ on MEP amplitude $(\% \mathrm{M})$. Post-hoc analysis showed a significant difference between the immobilized and free sides of patients (APB $P=0.01$; FCR $P=0.02$ ) and between the immobilized and left sides of controls (APB $P=0.0007$; FCR $P=0.004$ ). No differences were detected between the free sides of patients and both sides of controls, though there was a tendency towards slightly greater MEPs on the free sides of patients with both muscles. MEP amplitude was greater than the normal range (mean \pm 2 SD of control values) in all the immobilized sides. Abnormally high values were found in 8 patients in APB muscle, and in all patients in FCR muscle at low intensity $\left(120 \%\right.$ RMT, MEP $\left._{120}\right)$. Values were abnormal in 6 patients in APB muscle and in all patients in FCR muscle at high intensity (150\% RMT, $\left.\mathrm{MEP}_{150}\right)$.

During slight voluntary contraction no significant difference in MEP amplitude was found between the immobilized and free sides of patients or between any side of patients and controls (Fig. 2B and D).

\subsection{Paired pulse TMS}

Baseline MEP amplitude did not differ significantly between immobilized sides $(763 \pm 202 \mu \mathrm{V})$, free sides of patients $(702 \pm 218 \mu \mathrm{V})$ and controls (left sides $748 \pm 234 \mu \mathrm{V}$; right sides $789 \pm 256 \mu \mathrm{V})$. Repeated measures two-way ANOVA (between-subjects variable: side; within-subjects variable: ISI) showed a significant effect of the side [APB $F(3,38)=23.1, P=0.0001$, Fig. 3A; FCR $F(3,38)=11.8, P=0.007$, Fig. 3C) on conditioned MEP amplitude. Post-hoc analysis showed a significant difference between immobilized and free sides of

Table 1

CMAP amplitude, RMT and MEP latency (mean \pm SD) for APB and FCR muscle

\begin{tabular}{|c|c|c|c|c|c|c|}
\hline & \multicolumn{3}{|l|}{ APB muscle } & \multicolumn{3}{|l|}{ FCR muscle } \\
\hline & CMAP (mV) & RMT (\%) & MEP latency (ms) & CMAP (mV) & RMT (\%) & MEP latency (ms) \\
\hline Normal controls ${ }^{\mathrm{a}}$ & $15.2 \pm 3.4$ & $45.6 \pm 6.5$ & $22.4 \pm 1.7$ & $4.1 \pm 1.7$ & $47.1 \pm 6.8$ & $17.1 \pm 2.2$ \\
\hline Right sides & $16.1 \pm 4.5$ & $45.2 \pm 7.2$ & $22.6 \pm 1.4$ & $4.5 \pm 2.1$ & $45.9 \pm 8.1$ & $16.4 \pm 2.8$ \\
\hline Left sides & $14.8 \pm 3.8$ & $46.1 \pm 6.9$ & $22.2 \pm 1.9$ & $3.8 \pm 2.8$ & $48.0 \pm 7.5$ & $17.5 \pm 2.0$ \\
\hline Normal sides & $14.5 \pm 2.9$ & $46.5 \pm 5.4$ & $22.0 \pm 1.5$ & $4.4 \pm 2.2$ & $47.2 \pm 6.3$ & $16.7 \pm 2.3$ \\
\hline Immobilized sides & $10.6 \pm 4.1^{\mathrm{b}}$ & $42.2 \pm 6.4^{\mathrm{b}}$ & $22.7 \pm 2.1$ & $3.3 \pm 2.6$ & $44.5 \pm 7.2$ & $17.1 \pm 1.8$ \\
\hline
\end{tabular}

RMT is expressed as \% of maximum stimulator output. Reported here is the latency of MEPs to $120 \%$ RMT.

a Average data of left and right sides together.

b Parameters significantly reduced on immobilized sides. 
Table 2

Motor cortical maps in APB and FCR muscle

\begin{tabular}{|c|c|c|c|c|c|c|c|c|}
\hline & \multicolumn{4}{|l|}{ APB muscle } & \multicolumn{4}{|l|}{ FCR muscle } \\
\hline & Area (positions) & Volume (\%M) & $\operatorname{Cog}-\mathrm{x}(\mathrm{cm})$ & Cog-y $(\mathrm{cm})$ & Area (positions) & Volume (\%M) & $\operatorname{Cog}-\mathrm{x}(\mathrm{cm})$ & Cog-y $(\mathrm{cm})$ \\
\hline \multicolumn{9}{|l|}{ Normal controls } \\
\hline Right sides & $58.6 \pm 16.2$ & $543 \pm 356$ & $5.1 \pm 8.5$ & $0.1 \pm 0.7$ & $50.1 \pm 11.5$ & $321 \pm 206$ & $4.2 \pm 7.7$ & $0.2 \pm 0.6$ \\
\hline Left sides & $56.1 \pm 15.3$ & $485 \pm 397$ & $5.5 \pm 7.9$ & $-0.1 \pm 0.6$ & $51.8 \pm 12.8$ & $301 \pm 254$ & $4.1 \pm 8.1$ & $0.1 \pm 1.0$ \\
\hline Free sides & $56.2 \pm 15.8$ & $478 \pm 315$ & $5.2 \pm 9.1$ & $0.2 \pm 0.8$ & $52.3 \pm 12.4$ & $279 \pm 258$ & $4.4 \pm 7.5$ & $0.3 \pm 0.9$ \\
\hline Immobilized sides & $57.4 \pm 17.1$ & $1221 \pm 992^{\mathrm{a}}$ & $5.8 \pm 8.0$ & $0.2 \pm 0.7$ & $50.5 \pm 11.2$ & $1003 \pm 403^{a}$ & $4.0 \pm 6.4$ & $0.1 \pm 0.7$ \\
\hline
\end{tabular}

RMT is expressed as \% of maximum stimulator output. Reported here is the latency of MEPs to $120 \%$ RMT.

a Parameters significantly larger on immobilized sides.

patients (APB $P=0.001$; FCR $P=0.008$ ) and between immobilized sides of patients and controls (APB $P=0.001$; FCR $P=0.01)$. At the inhibitory ISIs of $2-4 \mathrm{~ms}\left(\mathrm{ICI}_{2-4}\right)$, MEP amplitude was significantly greater on the immobilized sides (APB $81.3 \pm 53.2 \%$ of test MEP; FCR $89.4 \pm 37.8 \%$ ) compared to the free sides of patients (APB $25.1 \pm 12.5 \%, \quad P=0.008, \quad$ Fig. $3 \mathrm{~B} ; \quad$ FCR $48.8 \pm 16.1 \%, P=0.013$, Fig. 3D) and to normal controls (APB $30.9 \pm 13.7 \%, \quad P=0.006, \quad$ Fig. $3 \mathrm{~B} ; \quad$ FCR $45.7 \pm 13.5 \%, P=0.012$, Fig. 3D). Intracortical facilitation $\left(\mathrm{ICF}_{10-30}\right)$ was significantly enhanced only in APB muscle on the immobilized sides $(227.2 \pm 112.8 \%$ of test MEP) compared to the free sides of patients (128.1 $\pm 40.3 \%, P=0.007$, Fig. 3B) and to normal controls $(95.5 \pm 31.3 \%, P=0.008$, Fig. $3 \mathrm{~B}) . \mathrm{ICF}_{10-30}$ in FCR muscle was greater on the immobilized sides $(151.4 \pm 79.5 \%$ of test MEP) compared to the free sides $(103.7 \pm 49.8 \%)$ and to normal controls $(80.2 \pm 55.4 \%)$, but the difference failed to prove statistically significant (Fig. 3D).

\subsection{F waves}

No significant differences in $\mathrm{F}$ wave parameters were found between controls (persistence $72 \pm 16 \%$; minimum latency $26.5 \pm 2.1 \mathrm{~ms}$; amplitude $114 \pm 51 \mu \mathrm{V}$; area $471 \pm 213 \mu \mathrm{Vms} ;$ F/CMAP, $13.2 \pm 4.7 \%$ ), immobilized sides $\quad(70 \pm 19 \% ; \quad 25.7 \pm 2.4 \mathrm{~ms} ; \quad 117 \pm 49 \mu \mathrm{V}$; $518 \pm 205 \mu \mathrm{Vms} ; 14.7 \pm 5.1 \%)$ and normal sides $(75 \pm 15 \% ; \quad 25.5 \pm 1.9 \mathrm{~ms} ; \quad 99 \pm 53 \mu \mathrm{V}$; $545 \pm 245 \mu \mathrm{Vms} ; 13.9 \pm 6.4 \%)$.

\subsection{Follow-up}

Four out of nine patients were retested at $\mathrm{T} 1$. The remaining patients were lost for compliance problems. Digital dexterity normalized at T1 (T1 $15.9 \pm 3.2 \mathrm{~s}$; T0 $37.6 \pm 6.2 \mathrm{~s}, P<0.001)$. CMAP amplitude from APB muscle was greater at $\mathrm{T} 1(12.1 \pm 1.9 \mathrm{~V})$ than at $\mathrm{T} 0$ $(8.2 \pm 2.7 \mathrm{~V}, P=0.05)$. RMT was slightly greater at $\mathrm{T} 1$ (APB $44.5 \pm 7.5 ;$ FCR $47.1 \pm 5.8)$ than at $\mathrm{T} 0(\mathrm{APB}$
A

APB muscle

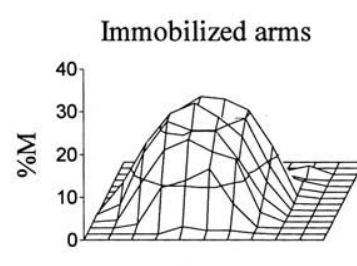

ANT.
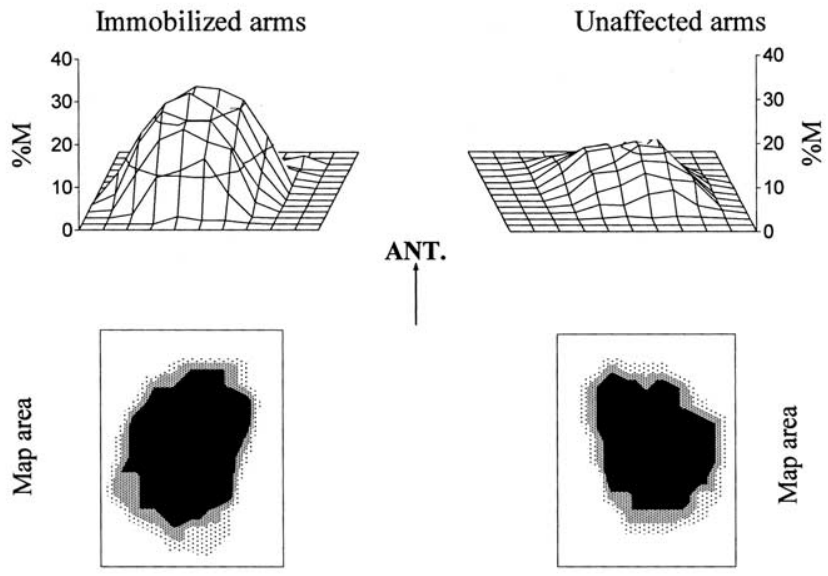

B

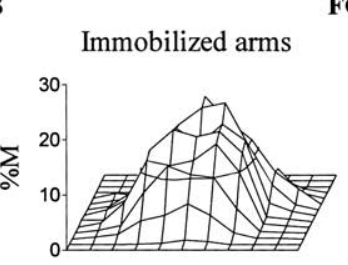

FCR muscle

Unaffected arms

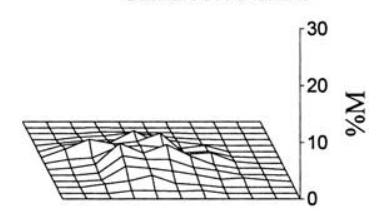

$\stackrel{\text { ANT. }}{\dagger}$
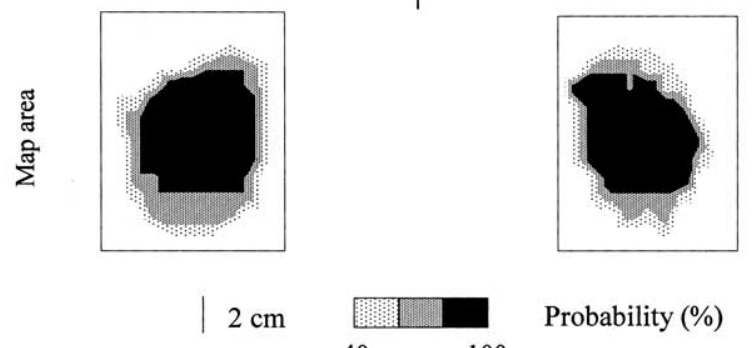

夰

Fig. 1. Volume and area of cortical motor maps in patients. The average volume of the motor maps is represented by a three-dimensional matrix where the cells contain the average of the $\% \mathrm{M}$ ( = \% CMAP) of the patients $(n=4)$. The average area of the map is represented by a two-dimensional matrix in which each cell contains the probability of recording MEP responses across all subjects. Both the APB (Panel A) and the FCR (Panel B) muscles showed a significant increase in volume on the immobilized sides, with no differences in area between the immobilized and free sides. 

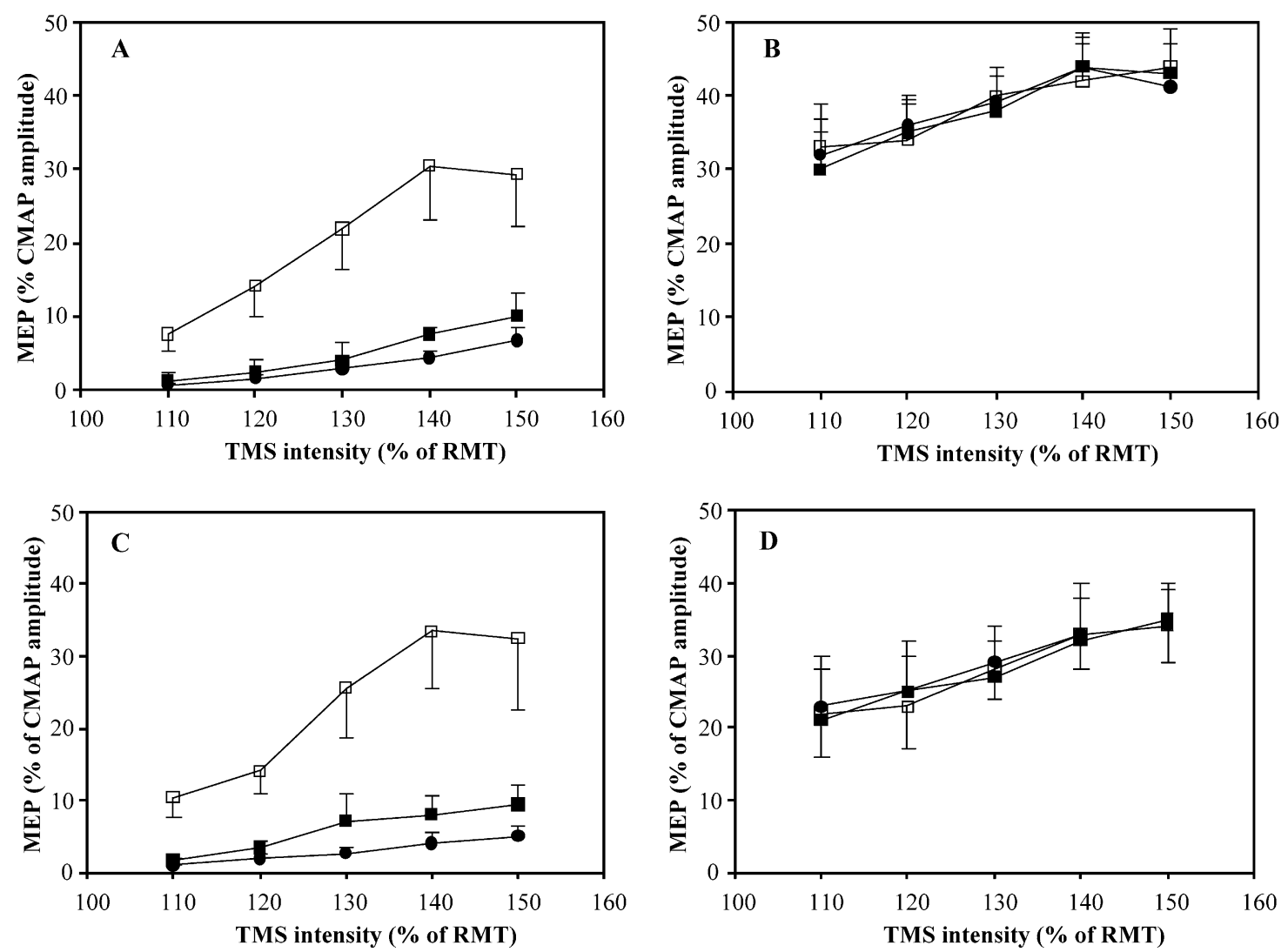

Fig. 2. MEP recruitment curves. Panels A and B, APB muscle. Panels C and D, FCR muscle. Panels A and C, Muscle rest. Panels B and D, Slight voluntary contraction. Filled circles, normal controls; filled boxes, patients' free sides; open boxes, patients' immobilized sides. Error bar, 1 SE. $X$-axis, TMS intensity (expressed as \% of RMT). $Y$-axis, MEP amplitude (expressed as \% of CMAP amplitude).

$42.8 \pm 6.2$; FCR $45.5 \pm 7.3$ ), but the difference was not statistically significant.

The MEP recruitment curve at rest tended to normalize at both low $\left(\mathrm{MEP}_{120}\right)$ and high intensity $\left(\mathrm{MEP}_{150}\right)$, though it reached normal values only in a few patients (Fig. 4A and $\mathrm{B})$. The difference between $\mathrm{T} 0$ and $\mathrm{T} 1$ was statistically significant for $\mathrm{MEP}_{120}$ (APB $P=0.012 \mathrm{FCR} P=0.043$ ) and $\mathrm{MEP}_{150}$ (APB $P=0.036$; FCR $P=0.048$ ).

Paired TMS data tended to normalize at $\mathrm{T} 1$, reaching the normal range only in a few patients (Fig. $4 \mathrm{C}$ and D). $\mathrm{ICI}_{2-4}$ decreased from $73.5 \pm 26.1 \%$ at $\mathrm{T} 0$ to $55.1 \pm 25.4 \%$ at $\mathrm{T} 1$ $(P=0.04)$ in APB muscle and from $90.4 \pm 18.5 \%$ at T0 to $67.9 \pm 16.2 \%$ at $\mathrm{T} 1(P=0.02)$ in FCR muscle. $\mathrm{ICF}_{10-30}$ decreased from $230.2 \pm 57.6 \%$ at $\mathrm{T} 0$ to $136.6 \pm 36.2 \%$ at $\mathrm{T} 1(P=0.04)$ in APB muscle and from $170.1 \pm 53.2 \%$ at T0 to $131.4 \pm 28.4 \%$ at T1 (ns) in FCR muscle.

No significant changes in any of the parameters were detected on the normal sides of patients at T1compared to T0.

\subsection{Correlation of hand dexterity and the duration of immobilization with motor excitability parameters}

No significant correlation was found between the duration of splinting and any TMS parameter. No significant correlation was found between digital dexterity and RMT, rest MEP recruitment curves, ICI and ICF for both TE and FCR muscles.

\section{Discussion}

This is the first report elucidating the mechanisms underlying motor reorganization in the representation of muscles undergoing sensorimotor restriction related to upper limb immobilization. The area and $\mathrm{CoG}$ of motor maps of immobilized muscles at rest did not change in our patients. Rather we found larger map volumes and steeper MEP recruitment curves at rest on the immobilized sides. MEP recruitment asymmetries disappeared during voluntary contraction. Imbalance between ICI and ICF, resulting in motor cortical hyperexcitability, was found in the cortex contralateral to the immobilized limbs. Even though some of the patients were lost in the follow-up, most of these abnormalities appear to be reversible, as they were largely normalized at the time of retesting. MEP recruitment curve and intracortical excitability measures were not correlated to digital dexterity in patients. These results suggest that hyperexcitability relies mainly on cortical mechanisms, but subcortical structures may contribute to the effect. 

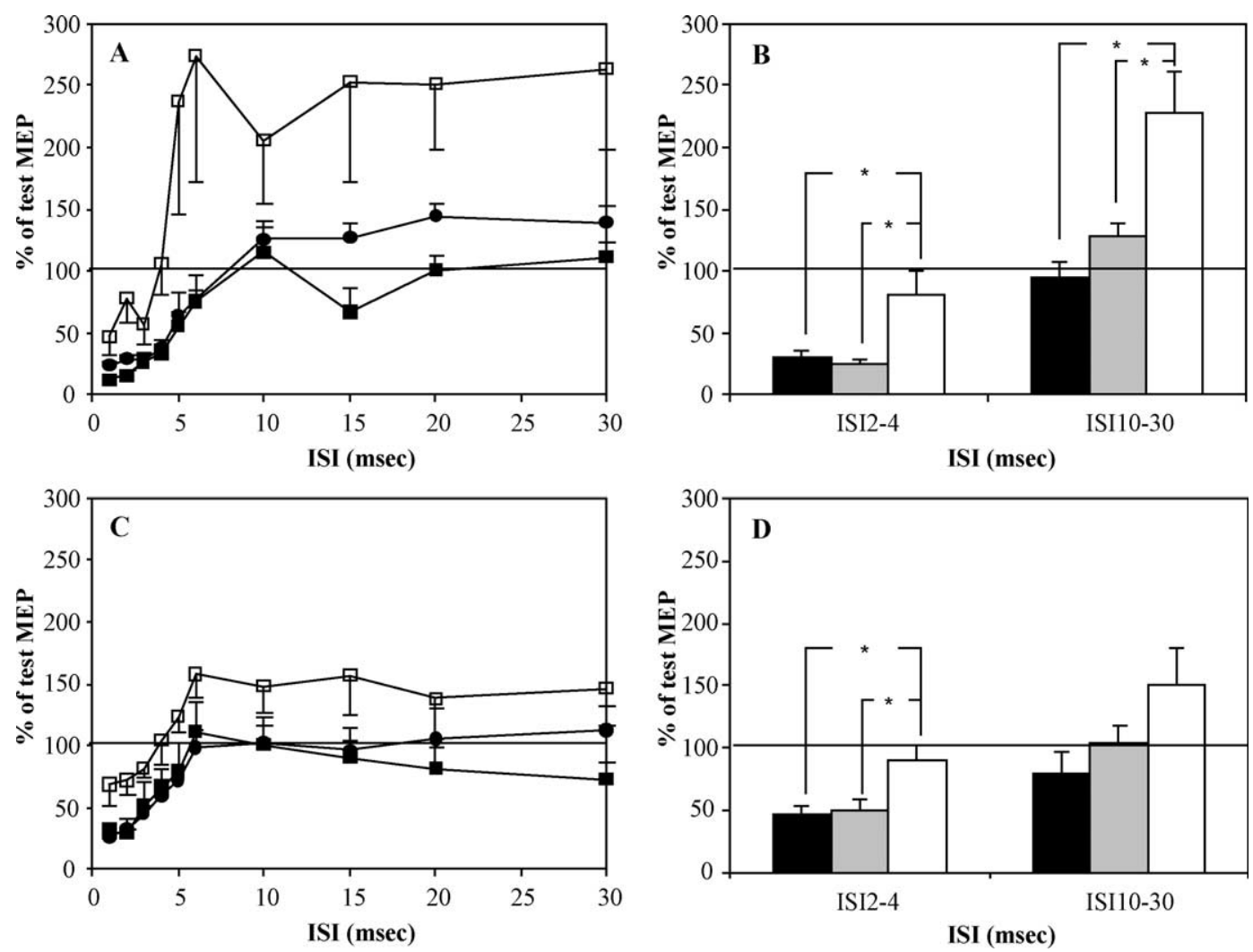

Fig. 3. Paired-TMS curves. Panels A and B, APB muscle. Panels C and D, FCR muscle. (A, C) Filled circles, normal controls; filled boxes, patients' free sides; open boxes, patients' immobilized sides. Horizontal continuous line (100\%) indicates the size of the unconditioned MEP. (B, D) Filled boxes, normal controls; shaded boxes, patients' free sides; open boxes, patients' immobilized sides. ${ }^{*}=P<0.01$ (Panel B); ${ }^{*}=P<0.02$ (Panel D). $X$-axis, interstimulus intervals (ms). $Y$-axis, MEP amplitude (expressed as \% of unconditioned MEP amplitude). Error bar, $1 \mathrm{SE}$.

\subsection{Motor maps and MEP recruitment curves}

The amplitude of CMAP was reduced in hand muscles on the immobilized sides, and a similar non-significant trend was found in forearm muscles. This finding, which is likely to be related to disuse atrophy, is in accordance with previous experimental reports of skeletal muscle changes after upper-limb immobilization (Desaphy et al., 2001; Seki et al 2001a; 2001b).

Our data, showing preservation of the borders of cortical maps of muscles undergoing sensorimotor restriction, are in agreement with the findings of a previous paper on subjects wearing upper limb splints (Zanette et al., 1997), as well as with recent data on the effect of autologous hand replantation for traumatic amputation (Röricht et al. (2001)). Motor map parameters during voluntary contraction were not tested in the present study, but previous data suggest that the abnormalities disappear during voluntary contraction (Zanette et al., 1997).

RMT and the slope of the MEP recruitment curve depend on the excitability properties of the whole corticospinal pathway, including any interneuronal relays (Devanne et al., 1997); this may be referred to as the strength of corticospinal projections. RMT was significantly lowered on the immobilized sides. This finding is at variance with previous data on immobilization. Zanette et al. (1997) reported a slight non-significant difference in RMT between the immobilized and the free sides of patients. Different hypotheses may be adduced to explain the discrepancy. The more likely are the more precise definition of the RMT in the present study, as TMS amplitude was varied in $1 \%$ steps, or the different type of coil used to determine RMT. Differences in the patients' population or in the immobilization duration may also contribute.

RMT mainly reflects membrane excitability in pyramidal neurons. One possible mechanism accounting for RMT lowering may involve changes in sodium channels (Chen et al., 1997; Ziemann et al., 1996), which have been implicated in some forms of plasticity (Halter et al., 1995). RMT was reduced after amputation (Chen et al., 1998; Cohen et al., 1991) but not after ischemic nerve block (Brasil-Neto et al., 1993; Ridding and Rothwell, 1997; Ziemann et al., 1998a), suggesting that changes in RMT may require long term deafferentation. This hypothesis is consistent with studies on rats with nerve lesions of different duration (Sanes et al., 1990). The MEP recruitment curve at rest measures the extent to which the alpha-motoneuronal pool is activated with increasing TMS intensities. The curve was steeper on the immobilized sides, mimicking the findings in amputees (Chen et al., 1998) or during ischemic 

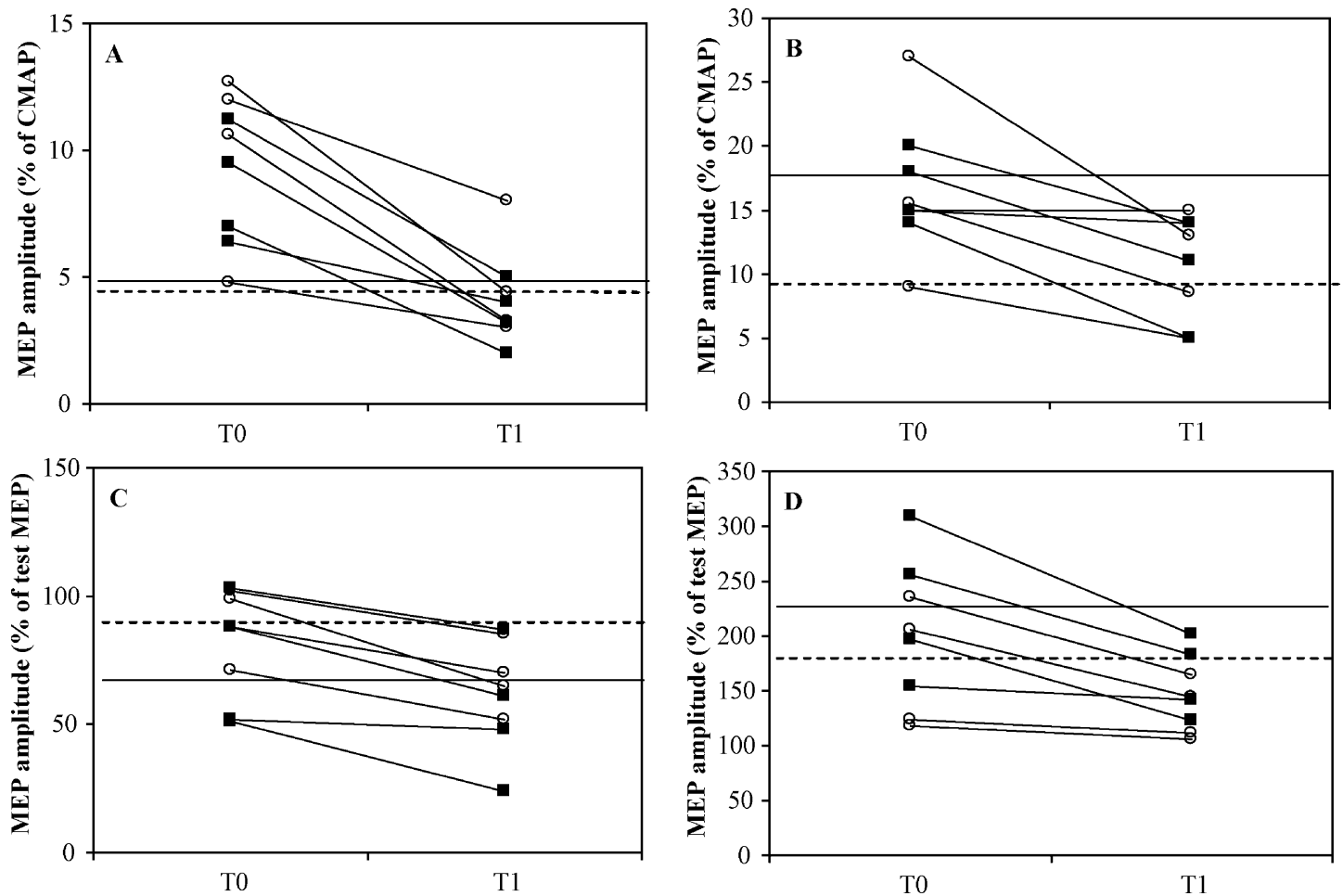

Fig. 4. Follow-up of MEP recruitment and paired TMS values. Represented here are the data from 4 patients' immobilized sides tested immediately after splint removal (T0) and 35-51 days later (T1). (A), low intensity TMS (MEP $\left.{ }_{120}\right)$. (B), high intensity TMS $\left(\mathrm{RMEP}_{150}\right)$. (C), intracortical inhibition (ICI $\left.{ }_{2-4}\right)$. (D), intracortical facilitation $\left(\mathrm{ICF}_{10-30}\right)$. Filled boxes, APB muscle; open circles, FCR muscle. Horizontal lines indicate the upper limit of normal controls $($ mean +2 SD) for APB muscle (continuous line) and FCR muscle (dashed line). $X$-axis, time. (A, B) $Y$-axis, MEP amplitude (expressed as $\%$ of CMAP). (C, D) $Y$-axis, MEP amplitude (expressed as $\%$ of test MEP).

anesthesia (Ridding and Rothwell, 1997). Factors influencing the curve are complex and interactive. They are mainly related to the amount of cortical elements recruited by TMS, the extent of temporal dispersion of the descending volleys and the strength of the synaptic input at spinal level; at present, little is known about the way these mechanisms interact in producing the stimulus-response relationship (Boroojerdi et al., 2001). It is likely that the lower RMT increases the size and number of descending volleys by prompting a substantial fringe of motoneurons, which are normally subthreshold for activation, to discharge in response to TMS. The lower RMT and the hyperexcitable stimulus-response curve resemble those observed during tonic voluntary contraction. This mechanism, however, is unlikely for a number of reasons. Audio and visual feedback were used to ensure muscle relaxation and MEP latencies were not reduced on the immobilized sides, confirming that the muscles were not activated. Moreover, ICF was normal or increased on the affected sides, while voluntary contraction reduces both ICI and ICF (Ridding et al., 1995). During voluntary contraction MEP curves were similar on the two sides of our patients. Similar results were found in a previous study on upper-limb amputees. MEP recruitment curve was steeper on the stump representation on the amputated side, but the asymmetry was not found during voluntary contraction (Ridding and Rothwell, 1997).

\subsection{Intracortical and spinal excitability}

ICI was reduced and ICF enhanced on the immobilized side as compared to the free side or to normal controls. Previous TMS studies documented the role of ICI reduction and ICF potentiation in neuroplasticity of stump muscles after upper limb (Schwenkreis et al., 2000) and lower limb amputation (Chen et al., 1998). Our data suggest that similar changes take place in the cortical representation of muscles exposed to sensorimotor restriction. Reduced ICI and enhanced ICF may depend on decreased GABAergic inhibition or increased NMDA-dependent excitatory activity (Schwenkreis et al., 2000; Ziemann et al., 1996; Ziemann et al., 1998b). Balance between ICI and ICF is necessary for the preservation of normal cortical representation and changes towards hyperexcitability are thought to play a major role in cortical plasticity (Jones, 1993).

F-wave parameters were normal on the immobilized sides, suggesting that, even though minimal changes may have gone undetected, spinal excitability is largely unchanged after immobilization. This finding is consistent with data on lower limb amputees (Chen et al., 1998).

ICI and ICF, recruitment curves and RMT tended to normalize in the course of follow-up. The incomplete reversal of the changes is compatible with experimental data (Jones, 1993) and might be explained by some kind of 
structural alterations in synaptic size or shape or the formation of new synapses (Darian-Smith and Gilbert, 1994; Kaas, 1991).

\subsection{Changes in the representation of unconstricted limb}

A slight non-significant potentiation of the MEP recruitment curve was detected in normal arms, with no significant changes in ICI, ICF or spinal excitability. Different explanations can be adduced to account for these findings. Hyperexcitability of the right cortex, projecting to the immobilized arm, might have been partially mirrored to the contralateral cortex, as a result of interhemispheric transfer of plasticity between corresponding cortical fields (Calford and Tweedale, 1990). Moreover, the functional inactivity of the right cortex might have resulted in a reduction of the inhibitory influences on the contralateral active areas (Hanajima et al., 2001). An alternative explanation is that the slight hyperexcitability of the projections to the free arm may depend on 'use-dependent' plasticity, because patients were increasingly dependent on the free right arm in everyday life. It has been suggested that such 'use-dependent' plasticity takes place in upper limb amputees (Elbert et al., 1997), but its relevance has not been confirmed by other authors (Schwenkreis et al., 2001).

\subsection{Mechanisms of motor cortex hyperexcitability}

Different hypotheses may be adduced to explain motor hyperexcitability. We suggest that a role may be played by changes in upper limb sensory feedback, which is quantitatively reduced and temporally more coherent after immobilization because of the simultaneous stimulation of larger territories during the forced posture (Coq and Xerri, 1999). Experimental data suggest that the coherence of sensory feedback is likely to play a role in modulating motor excitability. Hyperexcitability of the corticospinal projection to the hand muscles was found after prolonged synchronous stimulation of two nerves at the wrist (Ridding et al., 2001). Synchronous activation of upper limb muscles could induce changes in cortical motor maps, while no changes were found if the muscles were activated asynchronously (Cohen et al., 1995). What's more, deafferentation may enhance excitability at the higher centers, allowing the cortex to receive larger afferences to residual peripheral input (Millan, 1999). This hypothesis is in agreement with the hyperexcitable changes in the human visual cortex after light deprivation (Boroojerdi et al., 2000). An alternative or complementary hypothesis is that motor hyperexcitability may be a mechanism compensating for the reduced firing rate of motor units after immobilization (Seki et al., 2001a) to achieve optimal force generation.

Upper-limb splinting for wrist fracture does not represent a pure model of immobilization, because of the presence of inflammation and pain. It is unlikely that pain, which has been found to induce extensive neuroplastic reorganization (Birbaumer et al., 1997; Flor et al., 1995), may have contributed to motor hyperexcitability in our patients for a number of reasons. First, the duration of the pain was limited, and it ceased more than two weeks before TMS testing. What's more, previous data suggest that pain would inhibit, rather than enhance, motor cortex excitability (Farina et al., 2001; Le Pera et al., 2001). A recent TMS study reported motor cortex disinhibition in patients with complex regional pain syndrome of the hand (Schwenkreis et al., 2003), a clinical condition which represent a model of chronic pain related to neurogenic inflammation. These Authors reported bilateral reduction of ICI, while unilateral hyperexcitability to paired-TMS was found in our group of patients. Thus, the pattern of inflammation-related plasticity seems to differ from the present results. The role of pain may be of major importance in reconciling the apparently discrepant findings from the two previous studies on immobilization in humans. Zanette et al. (1997) reported enhanced motor excitability after upper limb immobilization in patients with short duration of pain (two-three weeks after wrist fracture). Liepert et al. (1995), in contrast, found reduced cortical maps of the leg muscles after ankle joint immobilization for complicated fractures of the distal tibia or talus; unfortunately, no mention of pain can be found in this study. One can hypothesize that the reduction in cortical representation of the leg muscles may be related to the persistence of pain at the time of mapping. Other factors accounting for this discrepancy may be the different duration of immobilization and the body part affected.

Maps of immobilized muscles were not reduced by invasion of the proximal muscles. This finding is easy to explain if one bears in mind that the proximal muscles, though free, were functionally restricted, due to reduced utilization of the entire upper limb. Experimental data suggest that sensory deprivation without competition yields only modest changes in cortical horizontal connectivity, which is believed to be important in shaping the borders of cortical maps (Finnerty and Connors, 2000).

It may be hypothesized that the volume-conducted MEPs from muscles responsible for distal movements may have contributed to the hyperexcitable changes, which were found in our patients. Even if the distal muscles were not completely immobilized, they were under-used in comparison to the corresponding muscles of the normal side because of the reduced activity of the whole upper-limb. What's more, we paid a great attention to record MEPs from APB and FCR muscles in order to avoid any volume-conducted contamination from surrounding muscles. These considerations suggest that, even if it cannot be completely excluded, the role of volume-conducted MEPs from near muscles appears to be marginal.

The finding that the MEP recruitment curve asymmetries disappear during muscle activation suggest the presence of a different level of 'rest' of the corticospinal system rather than true representational plasticity (Siebner and Rothwell, 
2003). The changes in the balance between ICI and ICF may indicate that a shift towards hyperexcitability takes place in the motor cortex of patients, who underwent upper-limb immobilization. The apparently paradoxical finding that similar hyperexcitable changes are brought about by practice, as in 'use-dependent' plasticity (Cohen et al., 1995) and sensorimotor restriction is similar to the observation that both anesthesia and painful stimulation may induce sensory hyperexcitability (Buchner et al., 2000). Enlargement of the perceived sizes of fingers has been reported after anesthesia, as well as repetitive stimulation and painful cooling of the digit (Gandevia and Phegan, 1999).

The effect of upper-limb immobilization yielded conflicting results in the field of movement disorders. It is known that segmental dystonia may develop after upper limb immobilization. Okun et al. (2002) reported a series of patients, who exhibited segmental dystonia following removal of a cast; only half of them experienced pain during casting. The Authors suggested that immobilization can induce dystonia, even in the absence of pain, because splinting may result in repetitive, spatially and temporally proximate, stereotyped, and attended sensory afferences. An intriguing point is that the hyperexcitable changes, which were found on immobilized sides of our patients, strongly resemble those found in the motor cortex of dystonic patients (Berardelli et al., 1998). These data seems to be in contrast with the observation that limb immobilization may ameliorate dystonic symptoms in patients with occupational dystonia (Priori et al., 2001). It may seem difficult to reconcile the observation that upper-limb immobilization may induce dystonia in some patients, while ameliorating dystonic symptoms in others. This point may be even more confusing, as some Authors reported that sensory training by learning to read Braille is an effective treatment for focal hand dystonia (Zeuner et al., 2002) and effective behavioral treatment for dystonia may reverse the abnormalities of cortical sensory maps (Candia et al., 2003). The effectiveness of two opposite behavioral strategies (i.e. inactivating the upper-limb and training the upper-limb in a difficult sensory learning task) in ameliorating dystonia may be reconciled by suggesting that neuroplastic changes may take place in very different settings, which involve sensorymotor retuning.

We were unable to find any significant correlation between cortical hyperexcitability and hand function in our patients, as some of them showed abnormal hand dexterity in spite of normal TMS parameters. We could only document that the partial reversal of motor hyperexcitability at T1 was associated with normalization of hand performance. Worse hand performances on the immobilized sides rely mainly upon peripheral factors, including stiffness of joint and muscles, effects of the injury and lack of motor practice. It is probable that motor hyperexcitability may represent a compensatory response in order to overcome bad hand dexterity. This hypothesis seems more likely as the motor cortex play a fundamental role in selective hand movements. An alternative less likely hypothesis is that motor cortex hyperexcitability 'per se' might contribute to the hand clumsiness. From this point of view, motor hyperexcitability to upper limb immobilization might be a new example of 'maladaptive' plasticity, which has been postulated as contributing to the genesis of dystonic symptoms (Byl et al., 1996), phantom limb pain (Flor et al., 1995) and tinnitus (Mühlnickel et al., 1998). At the moment, the relationship between neuroplastic changes and sensorimotor function has not been clearly established (Moore and Schady, 2000). Further studies will be needed in the future to better understand to which extent the changes in motor cortex excitability are related to the loss or gain of hand function and dexterity.

\section{Acknowledgements}

The authors wish to thank Mr Dino Volpato for his technical assistance, Dr Antonio Forgione for his help with the experimental recordings and Dr Nicos Giotakis for patient referral.

\section{References}

Berardelli A, Rothwell JC, Hallett M, Thompson PD, Manfredi M, Marsden CD. The pathophysiology of primary dystonia. Brain 1998;121: 1195-212.

Birbaumer N, Lutzenberger W, Montoya P, Larbig W, Unertl K, Topfner S, Grodd W, Taub E, Flor H. Effects of regional anaesthesia on phantom limb pain are mirrored in changes in cortical reorganization. J Neurosci 1997; 17:5503-8.

Boroojerdi B, Bushara KO, Corwell B, Immisch I, Battaglia F, Muellbacher W, Cohen Leonardo G. Enhanced excitability of the human visual cortex induced by short-term light deprivation. Cereb Cortex 2000;10: 529-34

Boroojerdi B, Battaglia F, Muellbacher W, Cohen LG. Mechanisms influencing stimulus-response properties of the human corticospinal system. Clin Neurophysiol 2001;112:931-7.

Brasil-Neto JP, Valls-Sole J, Pascual-Leone A, Cammarota A, Amassian VE, Cracco R, et al. Rapid modulation of human cortical motor outputs following ischaemic nerve block. Brain 1993;116:511-25.

Buchner H, Richrath P, Grünholz J, Noppeney T, Waberski D, Gobbelé R, Willmes K, Treede RD. Differential effect of pain and spatial attention on digit representation in the human primary somatosensory cortex. NeuroReport 2000;11:1289-93.

Byl NN, Merzenich MM, Jenkins WM. A primate genesis model of focal dystonia and repetitive strain injury: I. Learning-induced dedifferentiation of the representation of the hand in the primary somatosensory cortex in adult monkeys. Neurology 1996;47:508-20.

Calford MB, Tweedale R. Interhemispheric transfer of plasticity in the cerebral cortex. Science 1990;249:805-7.

Candia V, Wienbruch C, Elbert T, Rockstroh B, Ray W. Effective behavioral treatment of focal hand dystonia in musicians alters somatosensory cortical organization. Proc Natl Acad Sci USA 2003;100:7942-6.

Chen R, Samii A, Canos M, Wassermann EM, Hallett M. Effects of phenytoin on cortical excitability in humans. Neurology 1997;49:881-3.

Chen R, Corwell B, Yaseen Z, Hallett M, Cohen LG. Mechanisms of cortical reorganization in lower-limb amputees. J Neurosci 1998;18: 3443-50. 
Cohen LG, Bandinelli S, Findley TW, Hallett M. Motor reorganization after upper limb amputation in man. A study with focal magnetic stimulation. Brain 1991;114:615-27.

Cohen LG, Gerloff C, Ikoma K, Hallett M. Plasticity of motor cortex elicited by training of synchronous movements of hand and shoulder. Soc Neurosci Abstr 1995;21:517.

Coq JO, Xerri C. Tactile impoverishment and sensorimotor restriction deteriorate the forepaw cutaneous map in the primary somatosensory cortex of adult rats. Exp Brain Res 1999;129:518-31.

Darian-Smith C, Gilbert GD. Axonal sprouting accompanies functional reorganization in adult cat striate cortex. Nature 1994;68:737-40.

Desaphy JF, Pierno S, Léoty C, George Jr. AL, De Luca AM, Camerino DC. Skeletal muscle disuse induces fibre type-dependent enhancement of $\mathrm{Na}+$ channel expression. Brain 2001;124:1100-13.

Devanne H, Lavoie BA, Capaday C. Input-output properties and gain changes in the human corticospinal pathway. Exp Brain Res 1997;114: 329-38.

Elbert T, Pantev C, Wienbruch C, Rockstroh B, Taub E. Increased cortica representation of the fingers of the left hand in string players. Science 1995;270:305-7

Elbert T, Sterr A, Flor H, Rockstroh B, Knecht S, Pantev C, Wienbruch C, Taub E. Input-increase and input-decrease types of cortical reorganization after upper extremity amputation in humans. Exp Brain Res 1997;117:161-4.

Farina S, Valeriani M, Rosso T, Aglioti S, Tamburin S, Fiaschi A, Tinazzi M. Transient inhibition of the human motor cortex by capsaicininduced pain. A study with transcranial magnetic stimulation. Neurosci Lett 2001;314:97-101.

Finnerty GT, Connors BW. Sensory deprivation without competition yields modest alterations of short-term synaptic dynamics. Proc Natl Acad Sci USA 2000;97:12864-8.

Flor H, Elbert T, Knecht S, Wienbruch C, Pantev C, Birbaumer N, Larbig W, Taub E. Phantom-limb pain as a perceptual correlate of cortical reorganization following arm amputation. Nature 1995;375: 482-4.

Gandevia SC, Phegan CML. Perceptual distortions of the human body image produced by local anaesthesia, pain and cutaneous stimulation. J Physiol 1999;514:609-16.

Halter JA, Carp JS, Wolpaw JR. Operantly conditioned motoneuron plasticity: possible role of sodium channels. J Neurophysiol 1995;73: 867-71.

Hanajima R, Ugawa Y, Machii K, Mochizuki H, Terao Y, Enomoto H, Furubayashi T, Shiio Y, Uesugi H, Kanazawa I. Interhemispheric facilitation of the hand motor area in humans. J Physiol (London) 2001; 531:849-59.

Jones EG. GABAergic neurons and their role in cortical plasticity in primates. Cereb Cortex 1993;3:361-72.

Jones EG. Cortical and subcortical contribution to activity-dependent plasticity in primate somatosensory cortex. Annu Rev Neurosci 2000; 23:1-37.

Kaas JH. Plasticity of sensory and motor maps in adult mammals. Annu Rev Neurosci 1991;14:137-67.

Kew JJ, Ridding MC, Rothwell JC, Passingham RE, Leigh PN, Sooriakumaran S, Frackowiak RS, Brooks DJ. Reorganization of cortical blood flow and transcranial magnetic stimulation maps in human subjects after upper limb amputation. J Neurophysiol 1994;72: 2517-24.

Kujirai T, Caramia MD, Rothwell JC, Day BL, Thompson PD, Ferbert A, Wroe S, Asselman P, Marsden CD. Corticocortical inhibition in human motor cortex. J Physiol (London) 1993;471:501-19.

Langlet C, Canu MH, Falempin M. Short-term reorganization of the rat somatosensory cortex following hypodynamia-hypokinesia. Neurosci Lett 1999;266:145-8.

Le Pera D, Graven-Nielsen T, Valeriani M, Oliviero A, Di Lazzaro V, Tonali PA, Arendt-Nielsen L. Inhibition of motor system excitability at cortical and spinal level by tonic muscle pain. Clin Neurophysiol 2001; 112:1633-41.
Liepert J, Tegenthoff M, Malin JP. Changes of cortical motor area size during immobilization. Electroencephalogr Clin Neurophysiol 1995;97: 382-6.

Liepert J, Storch P, Fritsch A, Weiller C. Motor cortex disinhibition in acute stroke. Clin Neurophysiol 2000;111:671-6.

Millan MJ. The induction of pain: an integrative review. Prog Neurobiol 1999;57:1-164

Moore CE, Schady W. Investigation of the functional correlates of reorganization within the human somatosensory cortex. Brain 2000; 123:1883-95

Mühlnickel W, Elbert T, Taub E, Flor H. Reorganization of auditory cortex in tinnitus. Proc Natl Acad Sci USA 1998;95:10340-3.

Okun MS, Nadeau SE, Rossi F, Triggs WJ. Immobilization dystonia. J Neurol Sci 2002;201:79-83.

Oldfield RC. The assessment and analysis of handedness: the Edinburgh inventory. Neuropsychologia 1971;9:97-113.

Pascual-Leone A, Nguyet D, Cohen LG, Brasil-Neto JP, Cammarota A, Hallett M. Modulation of muscle responses evoked by transcranial magnetic stimulation during the acquisition of new fine motor skills. J Neurophysiol 1995;74:1037-45.

Priori A, Pesenti A, Cappellari A, Scarlato G, Barbieri S. Limb immobilization for the treatment of focal occupational dystonia. Neurology 2001;57:405-9.

Ridding MC, Rothwell JC. Stimulus/response curves as a method of measuring motor cortical excitability in man. Electroencephalogr Clin Neurophysiol 1997;105:340-4.

Ridding MC, Taylor JL, Rothwell JC. The effect of voluntary contraction on cortico-cortical inhibition in human motor cortex. J Physiol (London) 1995;487:541-8.

Ridding MC, McKay DR, Thompson PD, Miles TS. Changes in corticomotor representations induced by prolonged peripheral nerve stimulation in humans. Clin Neurophysiol 2001;112:1461-9.

Rijntjes M, Tegenthoff M, Liepert J, Leonhardt G, Kotterba S, Muller S, Kiebel S, Malin J-P, Diener H-C, Weiller C. Cortical reorganization in patients with facial palsy. Ann Neurol 1997;41:621-30.

Röricht S, Machetanz J, Irlbacher K, Niehaus L, Biemer E, Meyer BU. Reorganization of human motor cortex after hand replantation. Ann Neurol 2001;50:240-9.

Rossini P, Barker A, Berardelli A, Caramia M, Caruso G, Cracco RQ, et al. Non-invasive electrical and magnetic stimulation of the brain, spinal cord and roots: basic principles and procedures for routine clinical application. Report of IFCN committee. Electroencephalogr Clin Neurophysiol 1994;91:79-92.

Sanes JN, Donoghue JP. Plasticity and primary motor cortex. Annu Rev Neurosci 2000;23:393-415.

Sanes JN, Suner S, Donoghue JP. Dynamic organization of primary motor cortex output to target muscles in adult rats. I. Long-term patterns of reorganization following motor or mixed peripheral nerve lesions. Exp Brain Res 1990;79:479-91.

Schwenkreis P, Witscher K, Janssen F, Detwinkel R, Zenz M, Malin JP, Tegenthoff M. Changes of cortical excitability in patients with upper limb amputation. Neurosci Lett 2000;293:143-6.

Schwenkreis P, Witscher K, Janssen F, Pleger B, Dertwinkel R, Zenz M, Malin JP, Tegenthoff $M$. Assessment of reorganization in the sensorimotor cortex after upper limb amputation. Clin Neurophysiol 2001;112:627-35.

Schwenkreis P, Janssen MD, Rommel O, Pleger B, Völker B, Hosbach I, Dertwinkel R, Maier C, Tegenthoff M. Bilateral motor cortex disinhibition in complex regional pain syndrome (CRPS) type I of the hand. Neurology 2003;61:515-9.

Seki K, Taniguchi Y, Narusawa M. Effects of joint immobilization on firing rate modulation of human motor units. J Physiol 2001a;530:507-19.

Seki K, Taniguchi Y, Narusawa M. Alterations in contractile properties of human skeletal muscle induced by joint immobilization. J Physiol 2001b;530:521-32.

Siebner HR, Rothwell J. Transcranial magnetic stimulation: new insights into representational cortical plasticity. Exp Brain Res 2003;148:1-16. 
Taub E, Uswatte G, Elbert T. New treatment in neurorehabilitation founded on basic research. Nat Rev Neurosci 2002;3:228-36.

Tinazzi M, Zanette G, Volpato D, Testoni R, Bonato C, Manganotti P, Miniussi C, Fiaschi A. Neurophysiological evidence of neuroplasticity at multiple levels of the somatosensory system in patients with carpal tunnel syndrome. Brain 1998;121:1785-94.

Van der Kamp W, Maertens de Noordhout A, Thompson PD, Rothwell JC, Day BL, Marsden CD. Correlation of phasic muscle strength and corticomotoneuron conduction time in multiple sclerosis. Ann Neurol 1991;29:6-12.

Werhahn KJ, Fong JKY, Meyer BU, Priori A, Rothwell JC, Day BL, Thompson PD. The effect of magnetic coil orientation on the latency of surface EMG and single motor unit responses in the first dorsal interosseous muscle. Electroencephalogr Clin Neurophysiol 1994;93:138-46.

$\mathrm{Wu}$ CW, Kaas JH. Reorganization in primary motor cortex of primates with long-standing therapeutic amputations. J Neurosci 1999;19:7679-97.
Zanette G, Tinazzi M, Bonato C, di Summa A, Manganotti P, Polo A. Reversible changes of motor cortical outputs following immobilization of the upper limb. Electroencephalogr Clin Neurophysiol 1997;105: $269-79$.

Zeuner KE, Bara-Jimenez W, Noguchi PS, Goldstein SR, Dambrosia JM, Hallett M. Sensory training for patients with focal hand dystonia. Ann Neurol 2002;51:593-8.

Ziemann U, Lönnecker S, Steinhoff BJ, Paulus W. Effects of antiepileptic drugs on motor cortex excitability in humans: a transcranial magnetic stimulation study. Ann Neurol 1996;40:367-78.

Ziemann U, Corwell B, Cohen LG. Modulation of plasticity in human motor cortex after forearm ischemic nerve block. J Neurosci 1998a;18: $1115-23$.

Ziemann U, Hallett M, Cohen LG. Mechanisms of deafferentation-induced plasticity in human motor cortex. J Neurosci 1998b;18:7000-7. 